\title{
Attractors for Nonautonomous Parabolic Equations without Uniqueness
}

\author{
Cung The Anh ${ }^{1}$ and Nguyen Dinh Binh ${ }^{2}$ \\ ${ }^{1}$ Department of Mathematics, Hanoi National University of Education, 136 Xuan Thuy, Cau Giay, \\ 10307 Hanoi, Vietnam \\ ${ }^{2}$ Department of Applied Mathematics and Informatics, Hanoi University of Technology, 1 Dai Co Viet, \\ Hai Ba Trung, 10408 Hanoi, Vietnam
}

Correspondence should be addressed to Cung The Anh, anhctmath@hnue.edu.vn

Received 9 September 2009; Revised 12 February 2010; Accepted 8 April 2010

Academic Editor: Igor D. Chueshov

Copyright (c) 2010 C. The Anh and N. Dinh Binh. This is an open access article distributed under the Creative Commons Attribution License, which permits unrestricted use, distribution, and reproduction in any medium, provided the original work is properly cited.

\begin{abstract}
Using the theory of uniform global attractors of multivalued semiprocesses, we prove the existence of a uniform global attractor for a nonautonomous semilinear degenerate parabolic equation in which the conditions imposed on the nonlinearity provide the global existence of a weak solution, but not uniqueness. The Kneser property of solutions is also studied, and as a result we obtain the connectedness of the uniform global attractor.
\end{abstract}

\section{Introduction}

The understanding of the asymptotic behavior of dynamical systems is one of the most important problems of modern mathematical physics. One way to attack the problem for a dissipative dynamical system is to consider its global attractor. The existence of the global attractor has been derived for a large class of PDEs (see $[1,2]$ and references therein), for both autonomous and nonautonomous equations. However, these researches may not be applied to a wide class of problems, in which solutions may not be unique. Good examples of such systems are differential inclusions, variational inequalities, control infinite-dimensional systems, and also some partial differential equations for which solutions may not be known to be unique as, for example, some certain semilinear wave equations with high-power nonlinearities, the incompressible Navier-Stokes equation in three-space dimension, the Ginzburg-Landau equation, and so forth. For the qualitative analysis of the abovementioned systems from the point of view of the theory of dynamical systems, it is necessary to develop a corresponding theory for multivalued semigroups. 
In the last years, there have been some theories for which one can treat multivalued semiflows and their asymptotic behavior, including generalized semiflows theory of Ball [3], theory of trajectory attractors of Chepyzhov and Vishik [4], and theories of multivalued semiflows and semiprocesses of Melnik and Valero [5, 6]. Thanks to these theories, several results concerning attractors in the case of equations without uniqueness have been obtained recently for differential inclusion [5, 6], parabolic equations [7-9], the phase-field equation [10], the wave equation [11], the three-dimensional Navier-Stokes equation [3, 12], and so forth. On the other hand, when a problem does not possess the property of uniqueness, we have a set of solutions corresponding to each initial datum. We can speak then about a set of values attained by the solutions for every fixed moment of time. It is interesting to study the topological properties of such set and, in particular, its connectedness. This property is known as the Kneser property in the literature. The Kneser property has been studied for some parabolic equations [9, 13-15], semilinear wave equations [11], and so forth. By results in $[5,6]$, the Kneser property implies the connectedness of the global attractor. Although the existence of global attractor and the Kneser property have been derived for some classes of partial differential equations without uniqueness, to the best of our knowledge, little seems to be known for nonautonomous degenerate equations.

In this paper we study the following nonautonomous semilinear degenerate parabolic equation with variable, nonnegative coefficients, defined on a bounded domain $\Omega \subset \mathbb{R}^{N}, N \geq$ 2:

$$
\begin{gathered}
\frac{\partial u}{\partial t}-\operatorname{div}(\rho(x) \nabla u)+f(x, u)=g(x, t), \quad x \in \Omega, t>\tau, \\
\left.u\right|_{t=\tau}=u_{\tau}(x), \quad x \in \Omega, \\
\left.u\right|_{\partial \Omega}=0,
\end{gathered}
$$

where $u_{\tau} \in L^{2}(\Omega)$ is given, and the coefficient $\rho$, the nonlinearity $f$, and the external force $g$ satisfy the following conditions.

(H1) The function $\rho: \Omega \rightarrow \mathbb{R}$ satisfies $\rho \in L^{1}(\Omega)$ and, for some $\alpha \in(0,2), \lim _{x \rightarrow z} \inf \mid x-$ $\left.z\right|^{-\alpha} \rho(x)>0$ for every $z \in \bar{\Omega}$.

(H2) $f: \Omega \times \mathbb{R} \rightarrow \mathbb{R}$ is a Caratheodory function, that is, the function $f(\cdot, u)$ is measurable and the function $f(x, \cdot)$ is continuous, and satisfies

$$
\begin{gathered}
|f(x, u)| \leq C_{1}|u|^{p-1}+h_{1}(x), \quad \text { for some } p \geq 2, \\
u f(x, u) \geq C_{2}|u|^{p}-h_{2}(x)
\end{gathered}
$$

where $C_{1}, C_{2}$ are positive constants; $h_{1}, h_{2}$ are nonnegative functions such that $h_{1} \in L^{\infty}(\Omega)$ and $h_{2} \in L^{1}(\Omega)$ (see Remark 4.2 on a comment about conditions of $\left.h_{1}\right)$.

(H3) $g \in L_{b}^{2}\left(\mathbb{R}^{+}, L^{2}(\Omega)\right)$, where $L_{b}^{2}\left(\mathbb{R}^{+}, L^{2}(\Omega)\right)$ is the set of all translation-bounded functions (see Section 2.2 for its definition). 
The degeneracy of problem (1.1) is considered in the sense that the measurable, nonnegative diffusion coefficient $\rho(x)$ is allowed to have at most a finite number of (essential) zeroes at some points. The physical motivation of the assumption (H1) is related to the modelling of reaction diffusion processes in composite materials, occupying a bounded domain $\Omega$, in which at some points they behave as perfect insulator. Following [16, page 79 ], when at some points the medium is perfectly insulating, it is natural to assume that $\rho(x)$ vanishes at these points. Note that, in various diffusion processes, the equation involves diffusion of the type $\rho(x) \sim|x|^{\alpha}, \alpha \in(0,2)$.

In the autonomous case, which is the case $g$ independent of time $t$, the existence and long-time behavior of solutions to problem (1.1) have been studied in [17-20]. In this paper we continue studying the long-time behavior of solutions to problem (1.1) by allowing the external force $g$ to be dependent on time $t$. Moreover, the conditions imposed on the nonlinearity $f$ provide global existence of a weak solution to problem (1.1), but not uniqueness. Let $\boldsymbol{\Phi}_{\tau, \sigma}\left(u_{\tau}\right)$ be the set of all global weak solutions of problem (1.1) with the external force $g_{\sigma}$ instead of $g$ and initial datum $u(\tau)=u_{\tau}$. For each $\sigma \in \Sigma=\mathscr{\ell}_{w}(g)$, the closure of the set $\left\{g(\cdot+h) \mid h \in \mathbb{R}^{+}\right\}$in $L_{\mathrm{loc}}^{2}\left(\mathbb{R}^{+}, L^{2}(\Omega)\right)$ with the weak topology, we define the multivalued semiprocess $U_{\sigma}: \mathbb{R}_{d}^{+} \times L^{2}(\Omega) \mapsto 2^{L^{2}(\Omega)}$ as follows:

$$
U_{\sigma}\left(t, \tau, u_{\tau}\right)=\left\{u(t): u(\cdot) \in \Phi_{\tau, \sigma}\left(u_{\tau}\right)\right\}
$$

We prove that $U_{\sigma}$ is a strict multivalued semiprocess and then use the theory of multivalued semiprocesses of Melnik and Valero [6] to prove the existence of a uniform global compact attractor for the family of multivalued semiprocesses $\left\{U_{\sigma}\right\}_{\sigma \in \Sigma}$. Finally, following the general lines of the approach in $[9,11,14,15]$, we prove that the Kneser property holds for the set of all weak solutions, that is, the set of values attained by the solutions at every moment of time is connected. Thanks to the Kneser property, the uniform global attractor derived above is connected in $L^{2}(\Omega)$. We summarize our main results in the following theorem.

Theorem 1.1. Under conditions (H1)-(H3), problem (1.1) defines a family of strict multivalued semiprocesses $\left\{U_{\sigma}\right\}_{\sigma \in \Sigma}$, which possesses a uniform global compact connected attractor $A$ in $L^{2}(\Omega)$.

It is worth noticing that under some additional conditions on $f$, for example, $f_{u}^{\prime}(x, u) \geq$ $-C_{3}$ for all $x \in \Omega, u \in \mathbb{R}$, or a weaker assumption

$$
(f(x, u)-f(x, v))(u-v) \geqslant-C|u-v|^{2} \quad \forall x \in \Omega, u, v \in \mathbb{R}
$$

one can prove that the weak solution of problem (1.1) is unique. Then the multivalued semiprocess $U_{\sigma}$ turns to be a single-valued one and the uniform compact global attractor $\mathcal{A}$ derived in Theorem 1.1 is exactly the usual uniform attractor for the family of single-valued semiprocesses [1].

The rest of the paper is organized as follows. In Section 2, for convenience of readers, we recall some results on function spaces and uniform global attractors for multivalued semiprocesses. Section 3 is devoted to prove the global existence of a weak solution and the existence of a uniform global attractor of the family of multivalued semiprocesses associated to problem (1.1). In the last section, we prove the Kneser property for the solutions. As a result, we obtain the connectedness of the uniform global attractor. 


\section{Preliminaries}

\subsection{Function Space and Operator}

We recall some basic results on the function space which we will use. Let $N \geq 2, \alpha \in(0,2)$, and

$$
2_{\alpha}^{*}= \begin{cases}\frac{4}{\alpha} \in(2, \infty) & \text { if } N=2 \\ \frac{2 N}{N-2+\alpha} \in\left(2, \frac{2 N}{N-2}\right) & \text { if } N \geq 3\end{cases}
$$

The exponent $2_{\alpha}^{*}$ has the role of the critical exponent in the classical Sobolev embedding.

The natural energy space for problem (1.1) involves the space $\Phi_{0}^{1}(\Omega, \rho)$, defined as the closure of $C_{0}^{\infty}(\Omega)$ with respect to the norm

$$
\|u\|_{\Phi_{0}^{1}(\Omega, \rho)}:=\left(\int_{\Omega} \rho(x)|\nabla u|^{2} d x\right)^{1 / 2}
$$

The space $\Phi_{0}^{1}(\Omega, \rho)$ is a Hilbert space with respect to the scalar product

$$
(u, v):=\int_{\Omega} \rho(x) \nabla u \nabla v d x
$$

The following lemma comes from [21, Propositions 3.3-3.5].

Lemma 2.1. Assume that $\Omega$ is a bounded domain in $\mathbb{R}^{N}, N \geq 2$, and $\rho$ satisfies (H1). Then the following embeddings hold:

(i) $\Phi_{0}^{1}(\Omega, \rho) \hookrightarrow L^{2_{\alpha}^{*}}(\Omega)$ continuously,

(ii) $\Phi_{0}^{1}(\Omega, \rho) \hookrightarrow L^{p}(\Omega)$ compactly if $p \in\left[1,2_{\alpha}^{*}\right)$.

It is known (see [19]) that there exists a complete orthonormal system of eigenvectors $\left(e_{j}, \lambda_{j}\right)$ of the operator $A=\operatorname{div}(\rho(x) \nabla)$ such that

$$
\begin{gathered}
\left(e_{j}, e_{k}\right)=\delta_{j k}, \quad-\operatorname{div}\left(\rho(x) \nabla e_{j}\right)=\lambda_{j} e_{j}, \quad j, k=1,2, \ldots, \\
0<\lambda_{1} \leq \lambda_{2} \leq \lambda_{3} \leq \ldots, \quad \lambda_{j} \longrightarrow+\infty, \quad \text { as } j \longrightarrow \infty .
\end{gathered}
$$

\subsection{The Translation-Bounded Functions}

Definition 2.2. Let $\mathcal{\varepsilon}$ be a reflexive Banach space. A function $\varphi \in L_{\mathrm{loc}}^{2}\left(\mathbb{R}^{+}, \mathcal{\varepsilon}\right)$ is said to be translation-bounded if

$$
\|\varphi\|_{L_{b}^{2}}^{2}=\|\varphi\|_{L_{b}^{2}\left(\mathbb{R}^{+}, \varepsilon\right)}=\sup _{t \in \mathbb{R}^{+}} \int_{t}^{t+1}\|\varphi\|_{\varepsilon}^{2} d s<\infty .
$$


We will denote by $L_{b}^{2}\left(\mathbb{R}^{+}, \varepsilon\right)$ the set of all translation-bounded functions in $L_{\text {loc }}^{2}\left(\mathbb{R}^{+}, \varepsilon\right)$. Let $g \in L_{b}^{2}\left(\mathbb{R}^{+}, \mathcal{\varepsilon}\right)$ and $\mathscr{L}_{w}(g)$ be the closure of the set $\left\{g(\cdot+h) \mid h \in \mathbb{R}^{+}\right\}$in $L_{\text {loc }}^{2}\left(\mathbb{R}^{+}, \mathcal{\varepsilon}\right)$ with the weak topology. The following results are well-known.

Lemma 2.3 (see [1, Chapter 5, Proposition 4.2]). (1) For all $\sigma \in \mathscr{H}_{w}(g),\|\sigma\|_{L_{b}^{2}}^{2} \leq\|g\|_{L_{b}^{2}}^{2}$.

(2) The translation group $\{T(h)\}$ is weakly continuous on $\mathscr{H}_{w}(g)$.

(3) $T(h) \mathscr{d}_{w}(g) \subset \mathscr{d}_{w}(g)$ for $h \geq 0$.

(4) $\mathfrak{d}_{w}(g)$ is weakly compact.

\subsection{Uniform Attractors of Multivalued Semiprocesses}

Denote $\mathbb{R}_{d}^{+}=\{(t, \tau): 0 \leq \tau \leq t\}$. Let $X$ be a complete metric space, let $P(X)$ and $B(X)$ be the set of all nonempty subsets and the set of all nonempty bounded subsets of the space $X$, respectively, and let $\Sigma$ be a compact metric space.

Definition 2.4. The map $U: \mathbb{R}_{d}^{+} \times X \rightarrow D(X)$ is called a multivalued semiprocess (MSP) if

(1) $U(\tau, \tau, \cdot)=\operatorname{Id}($ the identity map)

(2) $U(t, \tau, x) \subset U(t, s, U(s, \tau, x))$, for all $x \in X, t, s, \tau \in \mathbb{R}^{+}, \tau \leq s \leq t$.

It is called a strict multivalued semiprocess if $U(t, \tau, x)=U(t, s, U(s, \tau, x))$.

We consider the family of MSP $\left\{U_{\sigma}\right\}_{\sigma \in \Sigma}$ and define the map $U_{\Sigma}: \mathbb{R}_{d}^{+} \times X \rightarrow D(X)$ by $U_{\Sigma}(t, \tau, x)=\bigcup_{\sigma \in \Sigma} U_{\sigma}(t, \tau, x)$, which is also a multivalued semiprocess. For $B \subset X$, denote

$$
\gamma_{T, \sigma}^{\tau}(B)=\cup_{t \geq T} U_{\sigma}(t, \tau, B)
$$

Definition 2.5. The family of MSP $\left\{U_{\sigma}\right\}_{\sigma \in \Sigma}$ is called uniformly asymptoticall upper semicompact if for any $B \in B(X)$ and $\tau \in \mathbb{R}_{+}$such that, for some $T=T(B, \tau), \gamma_{T, \Sigma}^{\tau}(B)=$ $\bigcup_{\sigma \in \Sigma} \gamma_{T, \sigma}^{\tau}(B) \in B(X)$, any sequence $\left\{\xi_{n}\right\}, \xi_{n} \in U_{\sigma_{n}}\left(t_{n}, \tau, B\right), \sigma_{n} \in \Sigma, t_{n} \rightarrow+\infty$, is precompact in $X$.

Definition 2.6. The family of MSP $\left\{U_{\sigma}\right\}_{\sigma \in \Sigma}$ is called pointwise dissipative if there exists $B_{0} \in$ $B(X)$ such that, for all $x \in X$,

$$
\operatorname{dist}\left(U_{\Sigma}(t, 0, x), B_{0}\right) \longrightarrow 0, \quad \text { as } t \longrightarrow \infty
$$

Definition 2.7. Let $X$ and $Y$ be two metric spaces. The multivalued map $F: X \rightarrow Y$ is said to be w-upper semicontinuous (w-u.s.c.) at $x_{0}$ if for any $\epsilon>0$ there exists $\delta>0$ such that

$$
F(x) \subset O_{\epsilon}\left(F\left(x_{0}\right)\right), \quad \forall x \in O_{\delta}\left(x_{0}\right) .
$$

The map $F$ is w-u.s.c. if it is w-u.s.c. at any $x \in D(F)=\{y \in X: F(x) \neq \emptyset\}$. 
Definition 2.8. The set $\mathbb{A}$ is called a uniform global attractor for the family of multivalued semiprocesses $U_{\Sigma}$ if the following are satisfied.

(1) It is negatively semiinvariant, that is, $\mathscr{A} \subset U_{\Sigma}(t, 0, \mathcal{A})$.

(2) It is uniformly attracting, that is, $\operatorname{dist}\left(U_{\Sigma}(t, \tau, B), A\right) \rightarrow 0$, as $t \rightarrow \infty$, for all $B \in$ $B(X)$ and $\tau \geq 0$.

(3) For any closed uniformly attracting set $Y$, we have $A \subset Y$ (minimality).

The following result comes from [6, Theorem 2] and [10, Theorem 3.12].

Theorem 2.9. Let $F\left(\mathbb{R}^{+}, Z\right)$ be a space of functions with values in $Z$, where $Z$ is a topological space, and let $\Sigma \subset F\left(\mathbb{R}^{+}, Z\right)$ be a compact metric space. Suppose that the family of multivalued semiprocesses $\left\{U_{\sigma}\right\}_{\sigma \in \Sigma}$ satisfies the following conditions.

(1) On $\Sigma$ is defined the continuous shift operator $T(s) \sigma(t)=\sigma(t+s), s \in \mathbb{R}^{+}$such that $T(h) \Sigma \subset \Sigma$, and for any $(t, \tau) \in \mathbb{R}_{d^{\prime}}^{+} \sigma \in \Sigma, s \in \mathbb{R}^{+}, x \in X$, one has

$$
U_{\sigma}(t+s, \tau+s, x)=U_{T(s) \sigma}(t, \tau, x) .
$$

(2) $U_{\sigma}$ is uniformly asymtopically upper semicompact.

(3) $U_{\sigma}$ is pointwise dissipative.

(4) The map $(x, \sigma) \mapsto U_{\sigma}(t, 0, x)$ has closed values and is w-upper semicontinuous.

Then the family of multivalued semiprocesses $\left\{U_{\sigma}\right\}_{\sigma \in \Sigma}$ has a uniform global compact attractor A. Moreover, if $\Sigma$ is a connected space, the map $(x, \sigma) \mapsto U_{\sigma}(t, 0, x)$ is upper semicontinuous with connected values and the global attractor $\mathbb{A}$ is contained in a connected bounded subset of $X$, then $A$ is a connected set.

\section{Existence of Uniform Global Attractors}

We denote

$$
\begin{gathered}
A=-\operatorname{div}(\rho(x) \nabla), \\
V=L^{2}\left(\tau, T ; \Phi_{0}^{1}(\Omega, \rho)\right) \cap L^{p}\left(\tau, T ; L^{p}(\Omega)\right), \\
V^{*}=L^{2}\left(\tau, T ; \Phi^{-1}(\Omega, \rho)\right)+L^{p^{\prime}}\left(\tau, T ; L^{p^{\prime}}(\Omega)\right),
\end{gathered}
$$

where $p^{\prime}$ is the conjugate index of $p$. In what follows, we assume that $u_{\tau} \in L^{2}(\Omega)$ is given.

Definition 3.1. A function $u(x, t)$ is called a weak solution of $(1.1)$ on $(\tau, T)$ if and only if

$$
\begin{gathered}
u \in V, \quad \frac{\partial u}{\partial t} \in V^{*}, \\
\left.u\right|_{t=\tau}=u_{\tau}, \quad \text { a.e. in } \Omega, \\
\int_{\tau}^{T}\left\langle u_{t}, \varphi\right\rangle+\int_{\tau}^{T} \int_{\Omega} \rho \nabla u \nabla \varphi+\int_{\tau}^{T}\langle f(x, u), \varphi\rangle=\int_{\tau}^{T}(g(t), \varphi),
\end{gathered}
$$

for all test functions $\varphi \in V$. 
It follows from Theorem 1.8 in [1, page 33] that if $u \in V$ and $d u / d t \in V^{*}$ then $u \in$ $C\left([\tau, T] ; L^{2}(\Omega)\right)$. This makes the initial condition in problem (1.1) meaningful.

Proposition 3.2. For any $\tau \in \mathbb{R}^{+}, T>\tau$, and $u_{\tau} \in L^{2}(\Omega)$ given, problem (1.1) has at least one weak solution on $(\tau, T)$.

Proof. The proof is classical, but we give some a priori estimates used later.

Consider the approximating solution $u_{n}(t)$ in the form

$$
u_{n}(t)=\sum_{k=1}^{n} u_{n k}(t) e_{k}
$$

where $\left\{e_{j}\right\}_{j=1}^{\infty}$ are the eigenvectors of the operator $A=-\operatorname{div}(\rho(x) \nabla)$. We get $u_{n}$ from solving the problem

$$
\begin{gathered}
\left\langle\frac{d u_{n}}{d t}, e_{k}\right\rangle+\left\langle A u_{n}, e_{k}\right\rangle+\left\langle f\left(x, u_{n}\right), e_{k}\right\rangle=\left(g, e_{k}\right), \\
\left(u_{n}(\tau), e_{k}\right)=\left(u_{\tau}, e_{k}\right), \quad k=1, \ldots, n .
\end{gathered}
$$

Using the Peano theorem, we get the local existence of $u_{n}$. We have

$$
\frac{1}{2} \frac{d}{d t}\left\|u_{n}\right\|_{L^{2}(\Omega)}^{2}+\left\|u_{n}\right\|_{\Phi_{0}^{1}(\Omega, \rho)}^{2}+\int_{\Omega} f\left(x, u_{n}\right) u_{n}=\int_{\Omega} g(t) u_{n}
$$

Using hypothesis (1.3) and the Cauchy inequality, we get

$$
\frac{1}{2} \frac{d}{d t}\left\|u_{n}\right\|_{L^{2}(\Omega)}^{2}+\left\|u_{n}\right\|_{\Phi_{0}^{1}(\Omega, \rho)}^{2}+C_{2}\left\|u_{n}\right\|_{L^{p}(\Omega)}^{p}-\left\|h_{2}\right\|_{L^{1}(\Omega)} \leq \frac{1}{2 \lambda_{1}}\|g(t)\|_{L^{2}(\Omega)}^{2}+\frac{\lambda_{1}}{2}\left\|u_{n}\right\|_{L^{2}(\Omega)}^{2}
$$

where $\lambda_{1}>0$ is the first eigenvalue of the operator $A$ in $\Omega$ with the homogeneous Dirichlet condition (noting that $\|u\|_{\Phi_{0}^{1}(\Omega, \rho)}^{2} \geq \lambda_{1}\|u\|_{L^{2}(\Omega)}^{2}$ ). Hence

$$
\frac{d}{d t}\left\|u_{n}\right\|_{L^{2}(\Omega)}^{2}+\left\|u_{n}\right\|_{\Phi_{0}^{1}(\Omega, \rho)}^{2}+2 C_{2}\left\|u_{n}\right\|_{L^{p}(\Omega)}^{p} \leq \frac{1}{\lambda_{1}}\|g(t)\|_{L^{2}(\Omega)}^{2}+2\left\|h_{2}\right\|_{L^{1}(\Omega)} .
$$

We show that the local solution $u_{n}$ can be extended to the interval $[\tau, \infty)$. Indeed, from (3.7) we have

$$
\frac{d}{d t}\left\|u_{n}\right\|_{L^{2}(\Omega)}^{2}+\lambda_{1}\left\|u_{n}\right\|_{L^{2}(\Omega)}^{2} \leq 2\left\|h_{2}\right\|_{L^{1}(\Omega)}+\frac{1}{\lambda_{1}}\|g(t)\|_{L^{2}(\Omega)}^{2} .
$$


By the Gronwall inequality, we obtain

$$
\begin{aligned}
& \left\|u_{n}(t)\right\|_{L^{2}(\Omega)}^{2} \\
& \quad \leqslant\left\|u_{n}(\tau)\right\|_{L^{2}(\Omega)}^{2} e^{-\lambda_{1}(t-\tau)}+\frac{2}{\lambda_{1}}\left(1-e^{-\lambda_{1}(t-\tau)}\right)\left\|h_{2}\right\|_{L^{1}(\Omega)}+\frac{1}{\lambda_{1}} \int_{\tau}^{t} e^{-\lambda_{1}(t-s)}\|g(s)\|_{L^{2}(\Omega)}^{2} d s \\
& \quad \leqslant\left\|u_{n}(\tau)\right\|_{L^{2}(\Omega)}^{2} e^{-\lambda_{1}(t-\tau)}+\frac{2}{\lambda_{1}}\left(1-e^{-\lambda_{1}(t-\tau)}\right)\left\|h_{2}\right\|_{L^{1}(\Omega)}+\frac{1}{\lambda_{1}\left(1-e^{-\lambda_{1}}\right)}\|g\|_{L_{b}^{2}}^{2}
\end{aligned}
$$

where we have used the fact that

$$
\begin{aligned}
\int_{\tau}^{t} e^{-\lambda_{1}(t-s)}\|g(s)\|_{L^{2}(\Omega)}^{2} d s & \leqslant \int_{t-1}^{t} e^{-\lambda_{1}(t-s)}\|g(s)\|_{L^{2}(\Omega)}^{2} d s+\int_{t-2}^{t-1} e^{-\lambda_{1}(t-s)}\|g(s)\|_{L^{2}(\Omega)}^{2} d s+\cdots \\
& \leqslant \int_{t-1}^{t}\|g(s)\|_{L^{2}(\Omega)}^{2} d s+e^{-\lambda_{1}} \int_{t-2}^{t-1}\|g(s)\|_{L^{2}(\Omega)}^{2} d s+\cdots \\
& \leqslant\left(1+e^{-\lambda_{1}}+e^{-2 \lambda_{1}}+\cdots\right)\|g\|_{L_{b}^{2}}^{2}=\frac{1}{1-e^{-\lambda_{1}}}\|g\|_{L_{b}^{2}}^{2}
\end{aligned}
$$

We now establish some a priori estimates for $u_{n}$. Integrating (3.7) on $[\tau, t], \tau<t \leq T$, we have

$$
\begin{aligned}
& \left\|u_{n}(t)\right\|_{L^{2}(\Omega)}^{2}+\int_{\tau}^{t}\left\|u_{n}(s)\right\|_{\Phi_{0}^{1}(\Omega, \rho)}^{2} d s+2 C_{2} \int_{\tau}^{t}\left\|u_{n}(s)\right\|_{L^{p}(\Omega)}^{p} d s \\
& \quad \leq\left\|u_{n}(\tau)\right\|_{L^{2}(\Omega)}^{2}+\frac{1}{\lambda_{1}} \int_{\tau}^{t}\|g(s)\|_{L^{2}(\Omega)}^{2} d s+2(t-\tau)\left\|h_{2}\right\|_{L^{1}(\Omega)} .
\end{aligned}
$$

The last inequality implies that

$$
\begin{gathered}
\left\{u_{n}\right\} \text { is bounded in } L^{\infty}\left(\tau, T ; L^{2}(\Omega)\right), \\
\left\{u_{n}\right\} \text { is bounded in } L^{2}\left(\tau, T ; \Phi_{0}^{1}(\Omega, \rho)\right), \\
\left\{u_{n}\right\} \text { is bounded in } L^{p}\left(\tau, T ; L^{p}(\Omega)\right) .
\end{gathered}
$$

Using hypothesis (1.2), one can prove that $\left\{f\left(x, u_{n}\right)\right\}$ is bounded in $L^{p^{\prime}}\left(\tau, T ; L^{p^{\prime}}(\Omega)\right)$. By rewriting the equation as

$$
\frac{d u_{n}}{d t}=-A u_{n}-f\left(x, u_{n}\right)+g
$$


we see that $\left\{d u_{n} / d t\right\}$ is bounded in $V^{*}$ and, therefore, in $L^{p^{\prime}}\left(\tau, T ; \Phi^{-1}(\Omega, \rho)+L^{p^{\prime}}(\Omega)\right)$. Therefore, we have

$$
\begin{gathered}
u_{n} \rightarrow u \quad \text { in } L^{2}\left(\tau, T ; \Phi_{0}^{1}(\Omega, \rho)\right), \\
\frac{d u_{n}}{d t}-\frac{d u}{d t} \quad \text { in } V^{*}, \\
f\left(x, u_{n}\right) \rightarrow \eta \quad \text { in } L^{p^{\prime}}\left(\tau, T ; L^{p^{\prime}}(\Omega)\right), \\
A u_{n} \rightarrow A u \quad \text { in } L^{2}\left(\tau, T ; \mathbb{\Phi}^{-1}(\Omega, \rho)\right),
\end{gathered}
$$

up to a subsequence. Hence by standard arguments [22, Chapter 1], one can show that $u$ is a weak solution of problem (1.1).

Denote by $\Phi_{\tau, \sigma}\left(u_{\tau}\right)$ the set of all global weak solutions (defined for $t \geq \tau$ ) of problem (1.1) with the external force $g_{\sigma}$ instead of $g$ and the initial datum $u(\tau)=u_{\tau}$. We put $\Sigma=$ $\mathscr{H}_{\omega}(g)$, so it is clear that $T(s) \Sigma \subset \Sigma$, where $T(s) \sigma=\sigma(\cdot+s)=g(\cdot+s), t \geq 0$, and that this map is continuous. For each $\sigma=g_{\sigma} \in \Sigma$, we define the map.

$$
U_{\sigma}\left(t, \tau, u_{\tau}\right)=\left\{u(t): u(\cdot) \in \Phi_{\tau, \sigma}\left(u_{\tau}\right)\right\}
$$

Lemma 3.3. $U_{\sigma}\left(t, \tau, u_{\tau}\right)$ is a strict multivalued semiprocess. Moreover,

$$
U_{\sigma}\left(t+s, \tau+s, u_{\tau}\right)=U_{T(s) \sigma}\left(t, \tau, u_{\tau}\right) \quad \forall u_{\tau} \in L^{2}(\Omega), s \geq 0, t \geq \tau \geq 0
$$

Proof. Given that $z \in U_{\sigma}\left(t, \tau, u_{\tau}\right)$, we have to prove that $z \in U_{\sigma}\left(t, s, U_{\sigma}\left(s, \tau, u_{\tau}\right)\right)$. Take $y(\cdot) \in$ $\Phi_{\tau, \sigma}\left(u_{\tau}\right)$ such that $y(\tau)=u_{\tau}$ and $y(t)=z$. Clearly, $y(s) \in U_{\sigma}\left(s, \tau, u_{\tau}\right)$. Then if we define $z(t)=y(t)$ for $t \geq s$, then we have $z(s)=y(s)$ and obviously $z(\cdot) \in \Phi_{s, \sigma}(y(s))$. Consequently, $z(t) \in U_{\sigma}\left(t, s, U_{\sigma}\left(s, \tau, u_{\tau}\right)\right)$.

Let now $z \in U_{\sigma}\left(t, s, U_{\sigma}\left(s, \tau, u_{\tau}\right)\right)$. Then there exist $u \in \Phi_{\tau, \sigma}\left(u_{\tau}\right)$ and $v \in \Phi_{s, \sigma}(u(s))$ such that $z=v(t)$. Define the function

$$
y(r)= \begin{cases}u(r) & \text { if } r \in[\tau, s], \\ v(r-s) & \text { if } r \in[s, t+s] .\end{cases}
$$

It is easy to see that $y \in \Phi_{\tau, \sigma}\left(u_{\tau}\right)$, so that $z=y(t+s) \in U_{\sigma}\left(t+s, \tau, u_{\tau}\right)$.

Let $z \in U_{\sigma}\left(t+s, \tau+s, u_{\tau}\right)$. Then there exists $u(\cdot) \in \Phi_{\tau+s, \sigma}\left(u_{\tau}\right)$ such that $z=u(t+s)$ and $v(\cdot)=u(\cdot+s) \in \boldsymbol{\Phi}_{\tau, T(s) \sigma}\left(u_{\tau}\right)$, so that $z=v(t) \in U_{T(s) \sigma}\left(t, \tau, u_{\tau}\right)$. Conversely, if $z \in U_{\tau, T(s) \sigma}\left(u_{\tau}\right)$, then there is $z \in \Phi_{\tau, T(s) \sigma}\left(u_{\tau}\right)$ such that $z=u(t)$ and $v(\cdot)=u(-s+\cdot) \in \Phi_{\tau+s, \sigma}\left(u_{\tau}\right)$ so that $z=v(t+s) \in U_{\sigma}\left(t+s, \tau+s, u_{\tau}\right)$.

Lemma 3.4. Let conditions (H1)-(H3) hold and let $\left\{u_{n}\right\} \subset \Phi_{\tau, \sigma_{n}}\left(u_{n}(\tau)\right)$ be an arbitrary sequence of solutions of (1.1) with initial data $u_{n}(\tau) \rightarrow \eta$ weakly in $L^{2}(\Omega)$ and external forces $g_{\sigma_{n}} \rightarrow g_{\sigma}$ in $\Sigma$. Then for any $T>\tau$ and $t_{n} \rightarrow t_{0}, t_{n}, t_{0} \in(\tau, T]$, there exists a subsequence such that $u_{n}\left(t_{n}\right) \rightarrow u\left(t_{0}\right)$ strongly in $L^{2}(\Omega)$, where $u(\cdot) \in \Phi_{\tau, \sigma}(\eta)$ is a weak solution of (1.1) with initial datum $u(\tau)=\eta$. 
Proof. Repeating the proof of inequality (3.11), we see that the solution $u_{n}$ satisfies

$$
\begin{aligned}
& \left\|u_{n}(t)\right\|_{L^{2}(\Omega)}^{2}+\int_{s}^{t}\left\|u_{n}(v)\right\|_{\Phi_{0}^{1}(\Omega, \rho)}^{2} d v+2 C_{2} \int_{S}^{t}\left\|u_{n}(v)\right\|_{L^{p}(\Omega)}^{p} d v \leq\left\|u_{n}(s)\right\|_{L^{2}(\Omega)}^{2} \\
& +\frac{1}{\lambda_{1}} \int_{s}^{t}\left\|g_{\sigma_{n}}(v)\right\|_{L^{2}(\Omega)}^{2} d v+2(t-s)\left\|h_{2}\right\|_{L^{1}(\Omega)}
\end{aligned}
$$

and a similar inequality holds for the solution $u$. Hence, by the arguments as in the proof of Proposition 3.2 and the Aubin-Lions lemma [22], we infer up to a subsequence that

$$
\begin{gathered}
u_{n} \rightarrow u \text { in } L^{2}\left(\tau, T ; \Phi_{0}^{1}(\Omega, \rho)\right), \\
u_{n} \longrightarrow u \text { in } L^{2}\left(\tau, T ; L^{2}(\Omega)\right), \\
u_{n}(t, x) \longrightarrow u(t, x) \text { for a.a. }(t, x) \in(\tau, T) \times \Omega, \\
u_{n}(t) \rightarrow u(t) \text { in } L^{2}(\Omega) \text { uniformly on }[\tau, T],
\end{gathered}
$$

where $u \in \Phi_{\tau, \sigma}(\eta)$.

Let now $t_{n} \rightarrow t_{0}$, with $t_{n}, t_{0} \in(\tau, T]$. We will prove that $u_{n}\left(t_{n}\right) \rightarrow u\left(t_{0}\right)$ strongly in $L^{2}(\Omega)$. Since $u_{n}\left(t_{n}\right) \rightarrow u\left(t_{0}\right)$ weakly in $L^{2}(\Omega)$, we have

$$
\left\|u\left(t_{0}\right)\right\|_{L^{2}(\Omega)}^{2} \leq \liminf _{n \rightarrow \infty}\left\|u_{n}\left(t_{n}\right)\right\|_{L^{2}(\Omega)}^{2}
$$

Thus, if we can show that $\limsup _{n \rightarrow \infty}\left\|u_{n}\left(t_{n}\right)\right\|_{L^{2}(\Omega)}^{2} \leq\left\|u\left(t_{0}\right)\right\|_{L^{2}(\Omega)}^{2}$, then the proof will be finished. It is easy to check that $u_{n}$ and $u$ satisfy the following inequalities:

$$
\begin{gathered}
\left\|u_{n}(t)\right\|_{L^{2}(\Omega)}^{2} \leq\left\|u_{n}(s)\right\|_{L^{2}(\Omega)}^{2}+2(t-s)\left\|h_{2}\right\|_{L^{1}(\Omega)}+\int_{s}^{t}\left(g_{\sigma_{n}}(v), u_{n}(v)\right) d v \\
\|u(t)\|_{L^{2}(\Omega)}^{2} \leq\|u(s)\|_{L^{2}(\Omega)}^{2}+2(t-s)\left\|h_{2}\right\|_{L^{1}(\Omega)}+\int_{s}^{t}\left(g_{\sigma}(v), u(v)\right) d s
\end{gathered}
$$

for all $t \geq s, t, s \in[\tau, T]$. Therefore, the functions

$$
\begin{gathered}
J_{n}(t)=\left\|u_{n}(t)\right\|_{L^{2}(\Omega)}^{2}-2 t\left\|h_{2}\right\|_{L^{1}(\Omega)}-\int_{\tau}^{t}\left(g_{\sigma_{n}}(s), u_{n}(s)\right) d s, \\
J(t)=\|u(t)\|_{L^{2}(\Omega)}^{2}-2 t\left\|h_{2}\right\|_{L^{1}(\Omega)}-\int_{\tau}^{t}\left(g_{\sigma}(s), u(s)\right) d s
\end{gathered}
$$

are continuous and nonincreasing on $[\tau, T]$. Moreover, $J_{n}(t) \rightarrow J(t)$ for a.a. $t \in(\tau, T)$.

We now prove that $\lim \sup J_{n}\left(t_{n}\right) \leq J\left(t_{0}\right)$, and this will imply that

$$
\underset{n \rightarrow \infty}{\limsup }\left\|u_{n}\left(t_{n}\right)\right\|_{L^{2}(\Omega)}^{2} \leq\left\|u\left(t_{0}\right)\right\|_{L^{2}(\Omega)}^{2}
$$


as desired. Indeed, suppose that $\left\{t_{m}\right\}$ is an increasing sequence in $\left(\tau, t_{0}\right)$ such that $J_{n}\left(t_{m}\right) \rightarrow$ $J\left(t_{m}\right)$ as $n \rightarrow \infty$. We can assume that $t_{m}<t_{n}$, so that

$$
J_{n}\left(t_{n}\right)-J\left(t_{0}\right) \leq J_{n}\left(t_{m}\right)-J\left(t_{0}\right) \leq\left|J_{n}\left(t_{m}\right)-J\left(t_{m}\right)\right|+\left|J\left(t_{m}\right)-J\left(t_{0}\right)\right| .
$$

Hence for any $\varepsilon>0$, there exist $t_{m}$ and $n_{0}\left(t_{m}\right)$ such that $J_{n}\left(t_{n}\right)-J\left(t_{0}\right) \leq \varepsilon$ for all $n \geq n_{0}$, and the result follows.

Theorem 3.5. Let conditions (H1)-(H3) hold. Then the family of multivalued semiprocesses $\left\{U_{\sigma}(t, \tau)\right\}_{\sigma \in \Sigma}$ has a uniform global compact attractor $A$ in $L^{2}(\Omega)$.

Proof. From (3.7), we obtain

$$
\frac{d}{d t}\|u(t)\|_{L^{2}(\Omega)}^{2}+\|u(t)\|_{\Phi_{0}^{1}(\Omega, \rho)}^{2}+2 C_{2}\|u(t)\|_{L^{p}(\Omega)}^{p} \leq \frac{1}{\lambda_{1}}\|g(t)\|_{L^{2}(\Omega)}^{2}+2\left\|h_{2}\right\|_{L^{1}(\Omega)} .
$$

Hence, similar to (3.9), we get

$$
\|u(t)\|_{L^{2}(\Omega)}^{2} \leqslant\|u(0)\|_{L^{2}(\Omega)}^{2} e^{-\lambda_{1} t}+\frac{2}{\lambda_{1}}\left(1-e^{-\lambda_{1} t}\right)\left\|h_{2}\right\|_{L^{1}(\Omega)}+\frac{1}{\lambda_{1}\left(1-e^{-\lambda_{1}}\right)}\|g\|_{L_{b}^{2}}^{2} .
$$

The last inequality implies that there is a positive constant $R$ such that

$$
\|u(t)\|_{L^{2}(\Omega)}^{2} \leq\|u(0)\|_{L^{2}(\Omega)}^{2} e^{-t}+R^{2} .
$$

Hence the ball $B_{0}=\left\{u \in L^{2}(\Omega):\|u\|_{L^{2}(\Omega)} \leq \sqrt{R^{2}+\epsilon}\right\}$ is an absorbing set for the map $(t, u) \mapsto$ $U_{\Sigma}(t, 0, u)$, that is, for any $B \in \mathcal{B}\left(L^{2}(\Omega)\right)$ there exists $T(B)$ such that $U_{\Sigma}(t, 0, B) \subset B_{0}$, for all $t \geq T(B)$.

We define now the set $K=\overline{U_{\Sigma}\left(1,0, B_{0}\right)}$. Lemma 3.4 implies that $K$ is compact. Moreover, since $B_{0}$ is absorbing, using Lemma 3.3 we have

$$
\begin{aligned}
U_{\sigma}(t, \tau, B) & =U_{\sigma}\left(t, t-1, U_{\sigma}(t-1, \tau, B)\right) \\
& =U_{T(t-1) \sigma}\left(1,0, U_{T(\tau) \sigma}(t-1-\tau, 0, B)\right) \subset U_{\Sigma}\left(1,0, B_{0}\right) \subset K,
\end{aligned}
$$

for all $\sigma \in \Sigma, B \in \mathcal{B}\left(L^{2}(\Omega)\right)$, and $t \geq T(B, \tau)$. It follows that any sequence $\left\{\xi_{n}\right\}$ such that $\left\{\xi_{n}\right\} \in$ $U_{\sigma_{n}}\left(t_{n}, \tau, B_{0}\right), \sigma_{n} \in \Sigma, t_{n} \rightarrow+\infty, B \in B\left(L^{2}(\Omega)\right)$, is precompact in $L^{2}(\Omega)$. It is a consequence of Lemma 3.4 that the map $U_{\sigma}$ has compact values for any $\sigma \in \Sigma$.

Finally, let us prove that the map $(\sigma, x) \mapsto U_{\sigma}(t, \tau, x)$ is upper semicontinuous for each fixed $t \geq \tau \geq 0$. Suppose that it is not true, that is, there exist $u_{0} \in L^{2}(\Omega), t \geq \tau \geq 0, \sigma_{0} \in \Sigma, \epsilon>0$, $\delta_{n} \rightarrow 0, u_{n} \in B_{\delta_{n}}\left(u_{0}\right), \sigma_{n} \rightarrow \sigma_{0}$, and $\xi_{n} \in U_{\sigma_{n}}\left(t, \tau, u_{n}\right)$ such that $\left\{\xi_{n}\right\} \notin B_{\epsilon}\left(U_{\sigma_{0}}\left(t, \tau, u_{0}\right)\right)$. But Lemma 3.4 implies (up to a subsequence) that $\xi_{n} \rightarrow \xi \in U_{\sigma_{0}}\left(t, \tau, u_{0}\right)$, which is a contradiction. The existence of the uniform global compact attractor follows then from Theorem 2.9. 


\section{The Kneser Property and Connectedness of the Attractors}

Let $\Phi_{\tau, T}\left(u_{\tau}\right)$ be the set of all weak solutions of the problem $(1.1)$ on $(\tau, T)$ with the initial datum $u(\tau)=u_{\tau}$. In this section we will check that the set

$$
K_{t}\left(u_{\tau}\right)=\left\{u(t): u(\cdot) \in \Phi_{\tau, T}\left(u_{\tau}\right)\right\}
$$

is connected in $L^{2}(\Omega)$.

We define a sequence of smooth functions $\psi_{k}: \mathbb{R}_{+} \rightarrow[0,1]$ satisfying

$$
\psi_{k}(s)= \begin{cases}1 & \text { if } 0 \leq s \leq k \\ 0 \leq \psi_{k}(s) \leq 1 & \text { if } k \leq s \leq k+1 \\ 0 & \text { if } s \geq k+1\end{cases}
$$

For every $k \geq 1$ we put $f_{k}(x, u)=\psi_{k}(|u|) f(x, u)+\left(1-\psi_{k}(|u|)\right)|u|^{p-2} u$. Then $f_{k}(x, \cdot) \in C(\mathbb{R}, \mathbb{R})$ and $\sup _{|u| \leq A}\left|f_{k}(x, u)-f(x, u)\right| \rightarrow 0$ as $k \rightarrow \infty$, for all $A>0$.

Let $\rho_{\epsilon}: \mathbb{R} \rightarrow \mathbb{R}$ be a mollifier, that is, $\rho_{\epsilon} \in C_{0}^{\infty}(\mathbb{R}, \mathbb{R})$ such that supp $\rho_{\epsilon} \subset B_{\epsilon}, \int_{\mathbb{R}} \rho_{\epsilon}(s) d s=$ $1, \rho_{\epsilon}(s) \geq 0$, for all $s \in \mathbb{R}$, where $B_{\epsilon}=\{u \in \mathbb{R}:|u| \leq \epsilon\}$.

We define the functions $f_{k}^{\epsilon}(x, u)=\int_{\mathbb{R}} \rho_{\epsilon}(s) f_{k}(x, u-s) d s$. Since, for any $k \geq 1, f_{k}(x,$. is uniformly continuous on $B_{k+1}$, there exists $\epsilon_{k} \in(0,1)$ such that, for any $u$ satisfying $|u| \leq k$ and for all $s$ for which $|u-s|<\epsilon_{k}$, we have

$$
\left|f_{k}(x, u)-f_{k}(x, s)\right| \leq \frac{1}{k}
$$

We put $f^{k}(x, u)=f_{k}^{\epsilon_{k}}(x, u)$. Then $f^{k}(x,.) \in C^{\infty}(\mathbb{R}, \mathbb{R})$, for all $k \geq 1$.

We now prove the following lemma.

Lemma 4.1. For all $k \geq 1$, the following statements hold:

$$
\begin{gathered}
\sup _{|u| \leq A}\left|f^{k}(x, u)-f(x, u)\right| \longrightarrow 0, \quad \text { as } k \longrightarrow \infty, \forall A>0, \\
\left|f^{k}(x, u)\right| \leq D_{1}|u|^{p-1}+D_{2}(x), \\
f^{k}(x, u) u \geq D_{3}|u|^{p}-D_{4}(x), \\
f_{u}^{k}(x, u) \geq-D_{5}(k), \quad \forall u \in \mathbb{R},
\end{gathered}
$$

where $D_{2} \in L^{\infty}(\Omega)$ and $D_{4} \in L^{1}(\Omega)$ are nonnegative functions, $D_{5}(k)$ is a nonnegative number, and the positive constants $D_{1}, D_{3}$ do not depend on $k$.

Proof. Because of (4.3), for any $u$ such that $|u|<k$, we have

$$
\left|f^{k}(x, u)-f_{k}(x, u)\right| \leq \int_{\mathbb{R}} \rho_{\epsilon_{k}}(u-s)\left|f_{k}(x, s)-f_{k}(x, u)\right| d s \leq \frac{1}{k}
$$


we obtain that for any $A>0$ and any $u$ such that $|u| \leq A$,

$$
\left|f^{k}(x, u)-f(x, u)\right| \leq\left|f^{k}(x, u)-f_{k}(x, u)\right|+\left|f_{k}(x, u)-f(x, u)\right|, \quad \forall k \geq A
$$

Hence (4.4) holds.

We prove that $f_{k}$ satisfies conditions type of (H2). Indeed, we have

$$
\begin{aligned}
f_{k}(x, u) u & =\psi_{k}(|u|) f(x, u) u+\left(1-\psi_{k}(|u|)\right)\left(|u|^{p-2} u\right) u \\
& \geq \psi_{k}(|u|)\left(C_{2}|u|^{p}-h_{2}(x)\right)+\left(1-\psi_{k}(|u|)\right)|u|^{p} \\
& \geq \tilde{C}_{2}|u|^{p}-\tilde{h}_{2}(x)
\end{aligned}
$$

where $\widetilde{C}_{2}=\min \left\{1, C_{2}\right\}, \tilde{h}_{2} \in L^{1}(\Omega)$, and

$$
\begin{aligned}
\left|f_{k}(x, u)\right| & \leq D\left(|f(x, u)|+\left.|| u\right|^{p-2} u \mid\right) \\
& \leq D\left(C_{1}|u|^{p-1}+h_{1}(x)+|u|^{p-1}\right) \\
& \leq D\left(C_{1}+1\right)|u|^{p-1}+D h_{1}(x),
\end{aligned}
$$

for some constant $D>0$. have

Now we check that $f^{k}$ satisfies (4.5) and (4.6). Using the above estimates for $f_{k}$, we

$$
\begin{aligned}
\left|f^{k}(x, u)\right| & =\left|\int_{\mathbb{R}} \rho_{\epsilon_{\mathrm{k}}}(s) f_{k}(x, u-s) d s\right| \\
& \leq \int_{\mathbb{R}} \rho_{\epsilon_{k}}(s)\left|f_{k}(x, u-s)\right| d s \\
& \leq \int_{\mathbb{R}} \rho_{\epsilon_{k}}(s)\left(D\left(C_{1}+1\right)|u-s|^{p-1}+D h_{1}(x)\right) d s \\
& \leq \int_{\mathbb{R}} \rho_{\epsilon_{k}}(s)\left(\widetilde{C}|u|^{p-1}+\widetilde{C}|s|^{p-1}+D h_{1}(x)\right) d s \\
& \leq \int_{\mathbb{R}} \rho_{\epsilon_{k}}(s)\left(\widetilde{C}|u|^{p-1}+\widetilde{C}\left|\epsilon_{k}\right|^{p-1}+D h_{1}(x)\right) d s \\
& \leq D_{1}|u|^{p-1}+D_{2}(x), \quad \text { where } D_{2} \in L^{\infty}(\Omega) .
\end{aligned}
$$


On the other hand, using Young's inequality and the estimates for $f_{k}$, we obtain

$$
\begin{aligned}
f^{k}(x, u) u & =\int_{\mathbb{R}} \rho_{\epsilon_{k}}(s) f_{k}(x, u-s)(u-s) d s+\int_{\mathbb{R}} \rho_{\epsilon_{k}}(s) f_{k}(x, u-s) s d s \\
& \geq \int_{\mathbb{R}} \rho_{\epsilon_{k}}(s)\left(\tilde{C}_{2}|u-s|^{p}-\tilde{h}_{2}(x)\right) d s-\int_{\mathbb{R}} \rho_{\epsilon_{k}}(s)\left(\frac{\widetilde{C}_{2}}{2 D\left(C_{1}+1\right)}\left|f_{k}(u-s)\right|^{p^{\prime}}+K_{0}|s|^{p}\right) d s \\
& \geq \int_{\mathbb{R}} \rho_{\epsilon_{k}}(s)\left(\tilde{C}_{2}|u-s|^{p}-\tilde{h}_{2}(x)\right) d s-\int_{\mathbb{R}} \rho_{\epsilon_{k}}(s)\left(\frac{\widetilde{C}_{2}}{2}|u-s|^{p}+\tilde{D} h_{1}^{p^{\prime}}(x)+K_{0}\left|\epsilon_{k}\right|^{p}\right) d s \\
& \geq \int_{\mathbb{R}} \rho_{\epsilon_{k}}(s) \frac{\widetilde{C}_{2}}{2}|u-s|^{p} d s-\tilde{h}_{2}(x)-\tilde{D} h_{1}^{p^{\prime}}(x)-\tilde{K}_{0} \\
& \geq D_{3}|u|^{p}-D_{4}(x), \quad \text { with } D_{4} \in L^{1}(\Omega)
\end{aligned}
$$

where in the last inequality we have used the fact that, for some $M>0$,

$$
|u|^{p}=|u-s+s|^{p} \leq M\left(|u-s|^{p}+|s|^{p}\right) \leq M\left(|u-s|^{p}+\epsilon_{k}^{p}\right) .
$$

Let us show that $f_{u}^{k}(x, u) \geq-C_{5}(k)$, for all $u \in \mathbb{R}$. Indeed, if $|u|>k+1$, we have

$$
f_{k u}(x, u)=\frac{\partial f_{k}(x, u)}{\partial u}=\frac{\partial\left(|u|^{p-2} u\right)}{\partial u}=(p-1)|u|^{p-2} \geq 0
$$

Then for $|u|>k+2$, we get

$$
f_{u}^{k}(x, u)=\int_{\mathbb{R}} \rho_{\epsilon_{k}}(s) f_{k u}(x, u) d s \geq 0 .
$$

Finally, if $|u| \leq k+2$, we have

$$
\left|f_{u}^{k}(x, u)\right| \leq \int_{\mathbb{R}}\left|\rho_{\epsilon_{k} u}(u-s)\right|\left|f_{k}(x, s)\right| d s \leq D_{5}(k)
$$

where we have used the above estimate for $f_{k}$ with noting that $h_{1} \in L^{\infty}(\Omega)$.

Remark 4.2. In fact, if we are concerned with the existence of the uniform global compact attractor in $L^{2}(\Omega)$, we only need to assume that $h_{1} \in L^{p^{\prime}}(\Omega)$. The stronger assumption, namely, $h_{1} \in L^{\infty}(\Omega)$, is used to prove (4.7), which is nessceary for the proof of the Kneser property and the connectedness of the uniform global attractor.

We are now in a position to prove the following theorem. 
Theorem 4.3. The set $K_{t}\left(u_{\tau}\right)$ is connected in $L^{2}(\Omega)$ for any $t \in[\tau, T]$.

Proof. The proof is quite standard (see, e.g., $[9,15]$ ), so we only give its sketch.

The case $t=\tau$ is obvious. Suppose then, that for some $t^{*} \in(\tau, T]$, the set $K_{t^{*}}\left(u_{\tau}\right)$ is not connected. Then there exist two compact sets $A_{1}, A_{2}$ in $L^{2}(\Omega)$ such that $A_{1} \cup A_{2}=K_{t^{*}}\left(u_{\tau}\right)$, $A_{1} \cap A_{2} \neq \emptyset$. Let $u_{1}, u_{2} \in \Phi_{\tau, T}\left(u_{\tau}\right)$ be such that $u_{1}\left(t^{*}\right) \in U_{1}, u_{2}\left(t^{*}\right) \in U_{2}$, where $U_{1}, U_{2}$ are disjoint open neighborhoods of $A_{1}, A_{2}$, respectively.

Let $u_{i}^{k}(t, \gamma), i=1,2$, be equal to $u_{i}(t)$ if $t \in[\tau, \gamma]$ and be a solution of the problem

$$
\begin{gathered}
\frac{\partial u}{\partial t}-\operatorname{div}(\sigma(x) \nabla u)+f^{k}(x, u)=g(t, x), \quad(t, x) \in(\gamma, T) \times \Omega, \\
\left.u\right|_{\partial \Omega}=0,\left.\quad u\right|_{t=\gamma}=u_{i}(\gamma, x)
\end{gathered}
$$

if $t \in[\gamma, T]$. Since Lemma 4.1 and Proposition 3.2, problem (4.18) has at least one weak solution. It follows from (4.7) that this solution is unique. Also, the maps $u_{i}^{k}(t, \gamma)$ are continuous on $\gamma$ for each fixed $k \geq 1$ and $t \in[\tau, T]$. For details of the proof of these facts, see, for example, [9]. Using (4.6), one can prove that the functions $u_{i}^{k}$ satisfy the estimate

$$
\begin{gathered}
\left\|u_{i}^{k}(t)\right\|_{L^{2}(\Omega)}^{2}+2 \int_{\gamma}^{t}\left\|u_{i}^{k}(s)\right\|_{\Phi_{0}^{1}(\Omega, \sigma)}^{2} d s+2 D_{3} \int_{\gamma}^{t}\left\|u_{i}^{k}(s)\right\|_{L^{p}(\Omega)}^{p} \\
\leq\left\|u_{i}^{k}(\gamma)\right\|_{L^{2}(\Omega)}^{2}+K\left(\int_{\gamma}^{t}\|g(s)\|_{L^{2}(\Omega)}^{2} d s+1\right) .
\end{gathered}
$$

Now we put

$$
\gamma(\lambda)= \begin{cases}\tau-(T-\tau) \lambda & \text { if } \lambda \in[-1,0] \\ \tau+(T-\tau) \lambda & \text { if } \lambda \in[0,1]\end{cases}
$$

and define the function

$$
\varphi^{k}(\lambda)(t)= \begin{cases}u_{1}^{k}(t, \gamma(\lambda)) & \text { if } \lambda \in[-1,0] \\ u_{2}^{k}(t, \gamma(\lambda)) & \text { if } \lambda \in[0,1]\end{cases}
$$

We have $\varphi^{k}(-1)(t)=u_{1}^{k}(t, T)=u_{1}(t), \varphi^{k}(1)(t)=u_{2}^{k}(t, T)=u_{2}(t)$. The map $\lambda \mapsto \varphi^{k}(\lambda)(t)$ is continuous for any fixed $k \geq 1, t \in[\tau, T]$ (note that $u_{1}^{k}(t, \tau)=u_{2}^{k}(t, \tau)$ ) and $\varphi^{k}(-1)\left(t^{*}\right) \in U_{1}$, $\varphi^{k}(1)\left(t^{*}\right) \in U_{2}$, so that there exists $\lambda_{k} \in[-1,1]$ such that $\varphi^{k}\left(\lambda_{k}\right)\left(t^{*}\right) \notin U_{1} \cup U_{2}$.

Denote $u^{k}(t)=\varphi^{k}\left(\lambda_{k}\right)(t)$. Note that, for each $k \geq 1$, we have either $u^{k}(t)=u_{1}^{k}\left(t, \gamma\left(\lambda_{k}\right)\right)$ or $u^{k}(t)=u_{2}^{k}\left(t, \gamma\left(\lambda_{k}\right)\right)$. For some subsequence it is equal to one of them; say $u_{1}^{k}\left(t, \gamma\left(\lambda_{k}\right)\right)$. Now we will consider the function $u_{1}^{k}\left(t, \gamma\left(\lambda_{k}\right)\right), t \in[\tau, T]$. We have

$$
u^{k}(t)= \begin{cases}u_{1}(t) & \text { if } t \in\left[\tau, \gamma\left(\lambda_{k}\right)\right] \\ u_{1}^{k}\left(t, \gamma\left(\lambda_{k}(t)\right)\right) & \text { if } t \in\left[\gamma\left(\lambda_{k}\right), T\right]\end{cases}
$$


where $\gamma\left(\lambda_{k}\right) \rightarrow \gamma_{0} \in[\tau, T]$. We define the function

$$
\tilde{f}^{k}(x, v(t))= \begin{cases}f(x, v(t)) & \text { if } t \in\left[\tau, \gamma\left(\lambda_{k}\right)\right] \\ f^{k}(x, v(t)) & \text { if } t \in\left(\gamma\left(\lambda_{k}\right), T\right]\end{cases}
$$

By the continuity, $u_{1}\left(\gamma\left(\lambda_{k}\right)\right) \rightarrow u_{1}\left(\gamma_{0}\right)$ as $k \rightarrow \infty$. Moreover, from (4.18), (4.5), and (4.6), one can show that $\left\{u^{k}\right\}$ is bounded in $V,\left\{\tilde{f}^{k}\left(x, u^{k}\right)\right\}$ is bounded in $L^{p^{\prime}}\left(\tau, T ; L^{p^{\prime}}(\Omega)\right)$, and, therefore, $\left\{d u^{k} / d t\right\}$ is bounded in $L^{p^{\prime}}\left(\tau, T ; V^{*}\right)$. By the Aubin-Lions lemma [22], we can prove that $u^{k}$ converges weakly to a weak solution $u$ of (1.1) satisfying $u(\tau)=u_{\tau}$.

Finally, we can prove also that $u^{k}\left(t^{*}\right) \rightarrow u\left(t^{*}\right)$ in $L^{2}(\Omega)$ (see again [9]). From this we immediately obtain that $u\left(t^{*}\right) \notin U_{1} \cup U_{2}$, which is a contradiction. This completes the proof.

Corollary 4.4. If $A \subset L^{2}(\Omega)$ is an arbitrary connected set, then

$$
K_{t}(A)=\left\{u(t): u(\cdot) \in \Phi_{\tau, T}(A)\right\} \subset L^{2}(\Omega)
$$

is connected.

Proof. The proof is similar to the one of Corollary 7 in [9], so we omit it.

We now give the proof of the main theorem.

Proof of Theorem 1.1. Under conditions $(H 1)-(H 3)$, we have shown the existence of the uniform global compact attractor $\mathcal{A}$ of the family of processes $\left\{U_{\sigma}\right\}_{\sigma \in \Sigma}$ in Theorem 3.5. In addition, $U_{\sigma}$ is upper semicontinuous and has the compact values. Moreover, $U_{\sigma}$ has the connected values since Theorem 4.3. Since both $L^{2}(\Omega)$ and $\Sigma$ are connected, $\mathscr{A}$ is connected in $L^{2}(\Omega)$.

\section{Acknowledgments}

The authors would like to thank the reviewers for their helpful comments and suggestions. This work was supported by the Vietnam National Foundation for Science and Technology Development (NAFOSTED).

\section{References}

[1] V. V. Chepyzhov and M. I. Vishik, Attractors for Equations of Mathematical Physics, vol. 49 of American Mathematical Society Colloquium Publications, American Mathematical Society, Providence, RI, USA, 2002.

[2] R. Temam, Infinite-Dimensional Dynamical Systems in Mechanics and Physics, vol. 68, Springer, New York, NY, USA, 2nd edition, 1997.

[3] J. M. Ball, "Continuity properties and global attractors of generalized semiflows and the NavierStokes equations," Journal of Nonlinear Science, vol. 7, no. 5, pp. 475-502, 1997.

[4] V. V. Chepyzhov and M. I. Vishik, "Evolution equations and their trajectory attractors," Journal de Mathématiques Pures et Appliquées, vol. 76, no. 10, pp. 913-964, 1997.

[5] V. S. Melnik and J. Valero, "On attractors of multivalued semi-flows and differential inclusions," SetValued Analysis, vol. 6, no. 1, pp. 83-111, 1998. 
[6] V. S. Melnik and J. Valero, "On global attractors of multivalued semiprocesses and nonautonomous evolution inclusions," Set-Valued Analysis, vol. 8, no. 4, pp. 375-403, 2000.

[7] A. V. Kapustyan, "Global attractors of a nonautonomous reaction-diffusion equation," Differential Equations, vol. 38, no. 10, pp. 1467-1471, 2002, translation from Differensial'nyeUravneniya, vol. 38, no. 10, pp. 1378-1381, 2002.

[8] O. V. Kapustyan and D. V. Shkundīn, "The global attractor of a nonlinear parabolic equation," Ukrainian Mathematical Journal, vol. 55, no. 4, pp. 446-455, 2003.

[9] J. Valero and A. Kapustyan, "On the connectedness and asymptotic behaviour of solutions of reactiondiffusion systems," Journal of Mathematical Analysis and Applications, vol. 323, no. 1, pp. 614-633, 2006.

[10] A. V. Kapustyan, V. S. Melnik, and J. Valero, "Attractors of multivalued dynamical processes generated by phase-field equations," International Journal of Bifurcation and Chaos, vol. 13, no. 7, pp. 1969-1983, 2003.

[11] J. M. Ball, "Global attractors for damped semilinear wave equations," Discrete and Continuous Dynamical Systems. Series A, vol. 10, no. 1-2, pp. 31-52, 2004.

[12] G. R. Sell, "Global attractors for the three-dimensional Navier-Stokes equations," Journal of Dynamics and Differential Equations, vol. 8, no. 1, pp. 1-33, 1996.

[13] T. Kaminogo, "Kneser families in infinite-dimensional spaces," Nonlinear Analysis: Theory, Methods $\mathcal{E}$ Applications, vol. 45, no. 5, pp. 613-627, 2001.

[14] J. Valero, "On the Kneser property for some parabolic problems," Topology and Its Applications, vol. 153, no. 5-6, pp. 975-989, 2005.

[15] A. V. Kapustyan and J. Valero, “On the Kneser property for the complex Ginzburg-Landau equation and the Lotka-Volterra system with diffusion," Journal of Mathematical Analysis and Applications, vol. 357, no. 1, pp. 254-272, 2009.

[16] R. Dautray and J.-L. Lions, Mathematical Analysis and Numerical Methods for Science and Technology: Volume I: Physical Origins and Classical Methods, Springer, Berlin, Germany, 1985.

[17] N. I. Karachalios and N. B. Zographopoulos, "Convergence towards attractors for a degenerate Ginzburg-Landau equation," Zeitschrift für Angewandte Mathematik und Physik, vol. 56, no. 1, pp. 1130, 2005.

[18] N. I. Karachalios and N. B. Zographopoulos, "On the dynamics of a degenerate parabolic equation: global bifurcation of stationary states and convergence," Calculus of Variations and Partial Differential Equations, vol. 25, no. 3, pp. 361-393, 2006.

[19] C. T. Anh and P. Q. Hung, "Global existence and long-time behavior of solutions to a class of degenerate parabolic equations," Annales Polonici Mathematici, vol. 93, no. 3, pp. 217-230, 2008.

[20] C. T. Anh, N. D. Binh, and L. T. Thuy, "On the global attractors for a class of semilinear degenerate parabolic equations," Annales Polonici Mathematici, vol. 98, no. 1, pp. 71-89, 2010.

[21] P. Caldiroli and R. Musina, "On a variational degenerate elliptic problem," Nonlinear Differential Equations and Applications, vol. 7, no. 2, pp. 187-199, 2000.

[22] J.-L. Lions, Quelques Méthodes de Résolution des Problémes aux Limites Non Linéaires, Dunod, Paris, France, 1969. 


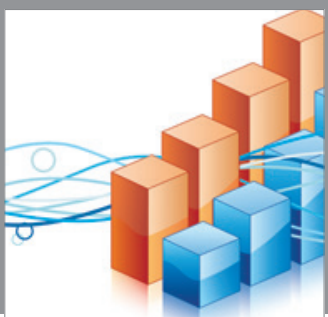

Advances in

Operations Research

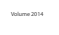

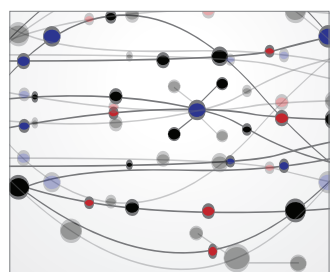

\section{The Scientific} World Journal
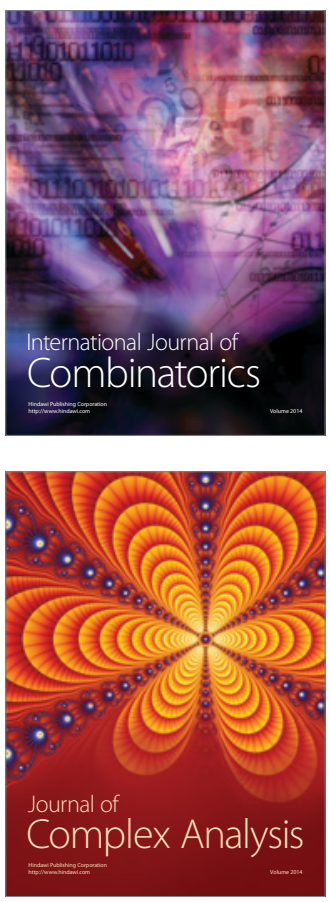

International Journal of

Mathematics and

Mathematical

Sciences
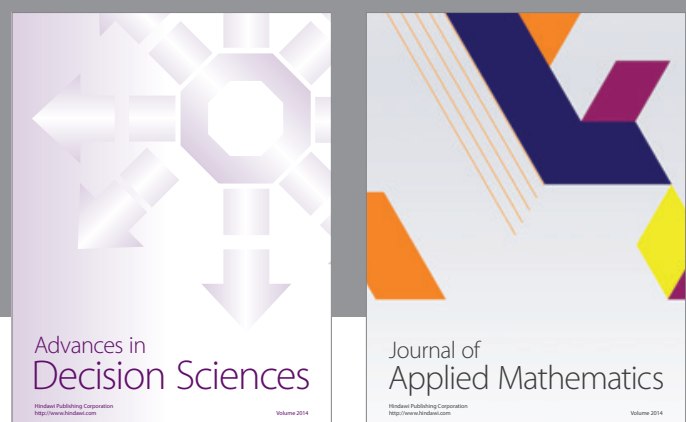

Journal of

Applied Mathematics
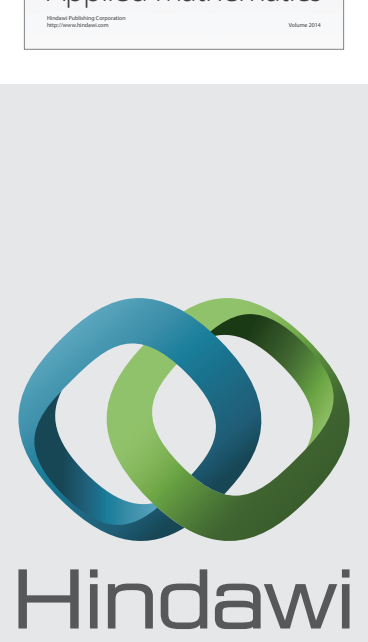

Submit your manuscripts at http://www.hindawi.com
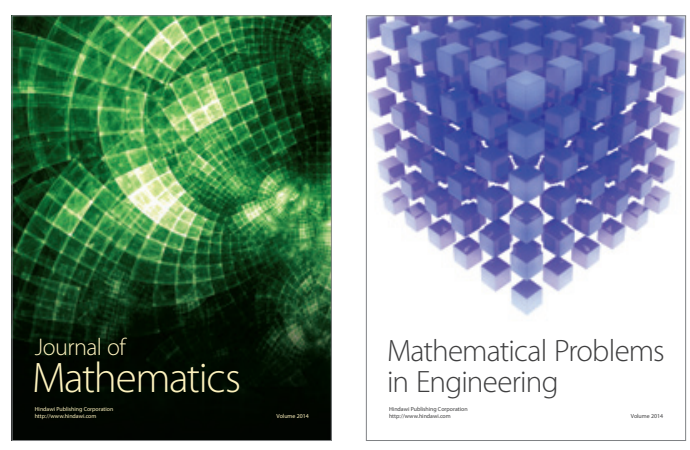

Mathematical Problems in Engineering
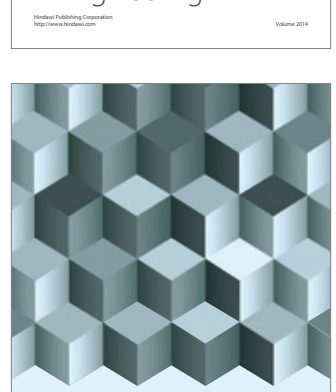

Journal of

Function Spaces
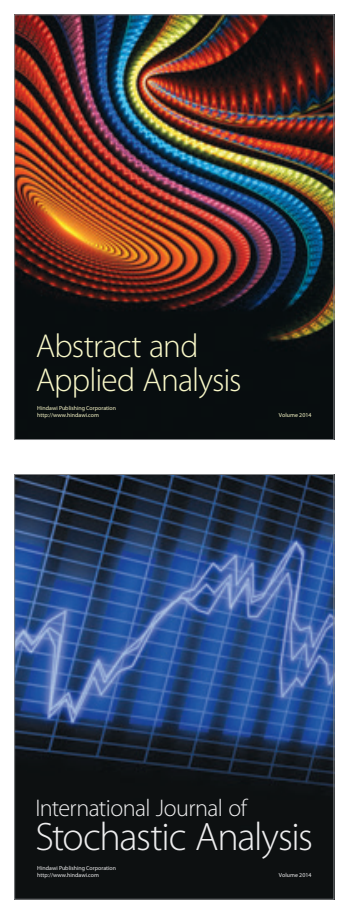

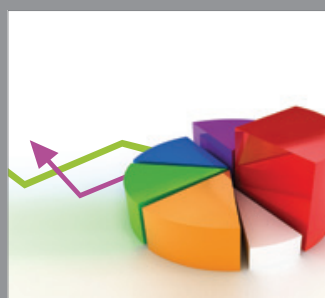

ournal of

Probability and Statistics

Promensencen
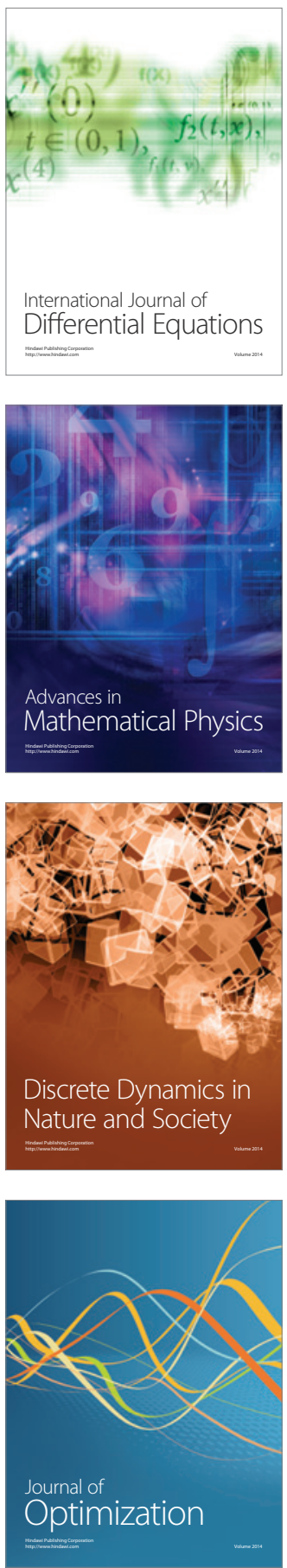